Int. J. Contemp. Math. Sciences, Vol. 2, 2007, no. 25, 1227 - 1236

\title{
Application of Homotopy Perturbation Method to the Fifth-Order Boundary Value Problems
}

\author{
Shaowei Shen \\ College of Statistics and Mathematics \\ Zhejiang Gongshang University \\ Hangzhou, 310018, P.R. China
}

\begin{abstract}
This paper deals with the fifth-order boundary value problems by using the homotopy perturbation method. This method provides the approximate solutions without discretization and the computation of the Adomian polynomials and the coefficients of sixth-degree B-spline functions. Numerical results show that this method is a promising and powerful tool for solving the fifth-order boundary value problems.
\end{abstract}

Mathematics Subject Classification: 34B05,34B15, 64D99

Keywords: Homotopy perturbation method; Fifth-order boundary value problems; Approximate solution; Analytical solution

\section{Introduction}

Consider the general fifth-order boundary value problem

$$
u^{(5)}(x)+f\left(x, u(x), u^{\prime}(x), u^{\prime \prime}(x), u^{\prime \prime \prime}(x), u^{(4)}(x)\right)=0,
$$

with the boundary conditions

$$
u(a)=\alpha_{0}, u^{\prime}(a)=\alpha_{1}, u^{\prime \prime}(a)=\alpha_{2}, u(b)=\beta_{0}, u^{\prime}(b)=\beta_{1},
$$

where $f$ is continuous function on $[a, b]$, and the parameters $\alpha_{i}, i=0,1,2$ and $\beta_{i}, i=0,1$ are real constants.

The fifth-order boundary value problems which arise in the mathematical modeling of viscoelastic flows and other branches of mathematical, physical and engineering sciences, have been widely studied by many authors $[5,6,8]$. The literature of numerical analysis contained little on the solution of the fifth-order boundary value problems[3]. The conditions for the existence and 
uniqueness of solutions of such problems were thoroughly discussed by R.P. Agarwal[2]. Two numerical algorithms namely spectral Galerkin methods and spectral collocation methods, were applied to address the numerical issues related to this type of problems[5, 6], respectively. Moreover, the fifth-order boundary value problems were investigated by M.S. Khan by using finite difference method [19], and considered by A.M. Wazwaz by means of Adomian decomposition method [24]. Recently, M. A. Khan et.al presented a class of methods based on non-polynomial sextic spline functions for the solution of a special fifth-order boundary-value problems [18], M. El-Gamel employed the Sinc-Galerkin method to solve the fifth-order boundary value problems [7]. We should point out that these approaches which were provided to solve this type of problems require a large amount of computational effort. In this paper, the homotopy perturbation method will be proposed to solve the fifth-order boundary value problems.

The homotopy perturbation method proposed by J.H. He [9, 10] is constantly being developed and applied to solve various nonlinear problems $[1,4$, $11,12,13,14,15,16,17,21,22,23]$. Unlike analytical perturbation methods, the homotopy perturbation method does not depend on a small parameter which is difficult to find. We focus on dealing with the fifth-order boundary value problems by means of the homotopy perturbation method. Two numerical examples will be presented to verify the efficiency of the homotopy perturbation method. Compared with the sixth-degree B-spline function method [3], the Adomian decomposition method [24] and Sinc-Galerkin method [7], the homotopy perturbation method provides a new approach to solve the fifth-order boundary value problems without discretization, Sinc interpolation and the computation of the Adomian polynomials. Therefore, this method is promising and readily implemented.

The rest of this paper is organized as follows. In section 2, we give the analysis of the homotopy perturbation method. In section 3, we present numerical results to demonstrate the efficiency of the homotopy perturbation method with the help of two examples.

\section{Analysis of the homotopy perturbation method}

To clarify the basic ideas of the homotopy perturbation method [13], we consider the following nonlinear differential equation

$$
A(u)-f(r)=0, r \in \Omega
$$

with boundary conditions

$$
B\left(u, \frac{d u}{d n}\right)=0, r \in \Gamma
$$


where $A$ is a general differential operator, $B$ is a boundary operator, $u$ is a known analytical function, and $\Gamma$ is the boundary of the domain $\Omega$.

The operator $A$ can be divided into two parts $L$ and $N$, where $L$ is linear, while $N$ is nonlinear. Therefore (3) can be rewritten as follows

$$
L(u)+N(u)-f(r)=0 .
$$

By the homotopy technique proposed by Liao [20], we can construct a homotopy $v(r, p): \Omega \times[0,1] \rightarrow R$ which satisfies

$$
H(v, p)=(1-p)\left[L(v)-L\left(u_{0}\right)\right]+p[A(v)-f(r)]=0,
$$

or

$$
H(v, p)=L(v)-L\left(u_{0}\right)+p L\left(u_{0}\right)+p[N(v)-f(r)]=0
$$

where $r \in \Gamma$ and $p \in[0,1]$ is an embedding parameter, $u_{0}$ is an initial approximation of (3), which satisfies the boundary conditions. By (6), it easily follows that

$$
\begin{aligned}
& H(v, 0)=L(v)-L\left(u_{0}\right)=0, \\
& H(v, 1)=A(v)-f(r)=0,
\end{aligned}
$$

and the changing process of $\mathrm{p}$ from zero to unity is just that of $H(v, p)$ from $L(v)-L\left(u_{0}\right)$ to $A(v)-f(r)$. In topology, this is called deformation, $L(v)-L\left(u_{0}\right)$ and $A(v)-f(r)$ are called homotopic.

The embedding parameter $p$ is introduced much more naturally, unaffected by artificial factors. Furthermore, it can be considered as a small parameter for $0<p \leq 1$. So it is very natural to assume that the solution of (6) can be expressed as

$$
v=v_{0}+p v_{1}+p^{2} v_{2}+\cdots
$$

Therefore, the approximate solution of (3) can be readily obtained as follows:

$$
u=\lim _{p \rightarrow 1} v=v_{0}+v_{1}+v_{2}+\cdots
$$

\section{$3 \quad$ Numerical examples}

In this section, two numerical examples will be presented to assess the efficiency of the homotopy perturbation method. For the sake of comparison, We will use the absolute error defined as

$$
\text { Errors }=\mid \text { analytical solution }- \text { approximate solution } \mid
$$


to verify the accuracy.

Example 1. Consider the following linear fifth-order boundary value problem

$$
u^{(5)}(x)=u-x(\cos x+\sin x)+5 \cos x,
$$

with the boundary conditions

$$
u(0)=0, u^{\prime}(0)=1, u^{\prime \prime}(0)=0, u\left(\frac{\pi}{2}\right)=0, u^{\prime}\left(\frac{\pi}{2}\right)=-\frac{\pi}{2} .
$$

The analytical solution of (13) is given by $u_{\text {anal. }}(x)=x \cos x$.

According to the homotopy perturbation method, we can construct the homotopy $\Omega \times[0,1] \rightarrow R$ which satisfies

$$
u^{(5)}(x)-u(x)-y_{0}^{(5)}(x)+p y_{0}^{(5)}(x)+p(x(\cos x+\sin x)-5 \cos x)=0,
$$

with the initial approximation $y_{0}(x)=x-\frac{1}{2} x^{3}+\frac{1}{24} x^{5}+A x^{6}+B x^{7}$, where A and $\mathrm{B}$ are unknown constants to be further determined.

Suppose the solution of (13) has the form:

$$
u=u_{0}+p u_{1}+\cdots .
$$

Substituting (16) into (15), and equating the terms of the same power of $p$, we have

$$
\begin{array}{ll}
p^{0}: & u_{0}^{(5)}(x)-u_{0}(x)-y_{0}^{(5)}(x)=0 \\
& y_{0}(x)=x-\frac{1}{2} x^{3}+\frac{1}{24} x^{5}+A x^{6}+B x^{7} \\
p^{1}: & u_{1}^{(5)}(x)-u_{1}(x)+x(\cos x+\sin x)-5 \cos x+y_{0}^{(5)}(x)=0 .
\end{array}
$$

By solving the above formulae results in the approximation $u(x)$. Then by the form of the solution (16) and the assumption $p=1$, we can obtain

$$
\begin{aligned}
u(x)= & 5-\frac{3}{2} x^{2}+\frac{1}{24} x^{4}+\frac{1}{720} x^{6}-\frac{1}{13440} x^{8}+\frac{1}{725760} x^{10} \\
& +\frac{1}{55440} A x^{11}+\frac{1}{95040} B x^{12}+(x-5) \cos (x)-x \sin (x) .
\end{aligned}
$$

Taking the Taylor series of $\cos (x)$ and $\sin (x)$, it follows that

$$
\begin{aligned}
u(x)= & x-\frac{1}{2} x^{3}+\frac{1}{24} x^{5}-\frac{1}{720} x^{7}+\frac{1}{40320} x^{9}+\frac{1}{479001600} x^{13} \\
& +\left(\frac{1}{55440} A-\frac{1}{3628800}\right) x^{11}+\left(\frac{1}{68428800}+\frac{1}{95040} B\right) x^{12}+O\left(x^{13}\right) .
\end{aligned}
$$


Using the boundary condition at $x=0$ and $x=1$ yields that

$$
A=0.000011418114, B=-0.001405532237 .
$$

Substituting (20) into (19), it follows that the approximate solution is given by

$$
\begin{aligned}
u(x)= & x-\frac{1}{2} x^{3}+\frac{1}{24} x^{5}-\frac{1}{720} x^{7}+\frac{1}{40320} x^{9}+\frac{1}{479001600} x^{13} \\
& -2.7536723780 \times 10^{-7} x^{11}-1.7511940353 \times 10^{-10} x^{12}+O\left(x^{13}\right) .
\end{aligned}
$$

Table 1 exhibits the analytical solutions, the approximate solutions obtained by the homotopy perturbation method, and the absolute errors. Obviously, the homotopy perturbation method provides high accuracy compared with the analytical solutions. In addition, reviewing sixth-degree B-spline function method [3] and the Adomian decomposition method [24] for solving this type of problems, the homotopy perturbation method gives the approximate solutions without discretization and the computation of the coefficients of sixth-degree B-spline functions and the Adomian polynomials. It's important to note that we get the high accuracy only by two components of the solution (16), and the accuracy can be further improved by considering more components of the solution (16).

Example 2. Consider the following nonlinear fifth-order boundary value problem [7]

$$
u^{(5)}(x)+u^{(4)}(x)+e^{-2 x} u^{2}(x)=2 e^{x}+1,
$$

with the boundary conditions

$$
u(0)=1, u^{\prime}(0)=1, u^{\prime \prime}(0)=1, u(1)=u^{\prime}(1)=e .
$$

The analytical solution of $(22)$ is given by $u_{\text {anal. }}(x)=e^{x}$.

Similarly, we can construct the homotopy $\Omega \times[0,1] \rightarrow R$ which satisfies

$$
u^{(5)}(x)-y_{0}^{(5)}(x)+p y_{0}^{(5)}(x)+p\left(u^{(4)}(x)+e^{-2 x} u^{2}(x)-2 e^{x}-1\right)=0,
$$

with the initial approximation $y_{0}(x)=1+x+\frac{1}{2} x^{2}+\frac{1}{6} A x^{3}+\frac{1}{24} B x^{4}$, where A and $\mathrm{B}$ are unknown constants to be further determined.

Consider the solution of $(22)$ as

$$
u=u_{0}+p u_{1}+\cdots .
$$

Applying the homotopy perturbation method, and rearranging based on powers of $p$ terms, we have

$$
\begin{array}{ll}
p^{0}: & u_{0}^{(5)}(x)-y_{0}^{(5)}(x)=0, y_{0}(x)=1+x+\frac{1}{2} x^{2}+\frac{1}{6} A x^{3}+\frac{1}{24} B x^{4}, \\
p^{1}: & u_{1}^{(5)}(x)+u_{0}^{(4)}(x)+e^{-2 x} u_{0}^{2}(x)-2 e^{x}-1+y_{0}^{(5)}(x)=0
\end{array}
$$


By choosing $u_{0}(x)=y_{0}(x)$, and solving the above formulae results in the approximation $u(x)$. According to (25) and the assumption $p=1$, we obtain

$$
\begin{aligned}
u(x)= & -\frac{211}{64}-\frac{245}{64} A-\frac{525}{256} A^{2}-\frac{2345}{512} B-\frac{5775}{1024} A B-\frac{17325}{4096} B^{2} \\
& +\left(\frac{217}{128}+\frac{115}{32} A+\frac{105}{64} A^{2}+\frac{245}{64} B+\frac{525}{128} A B+\frac{5775}{2048} B^{2}\right) x \\
& -\left(\frac{249}{128}+\frac{185}{128} A+\frac{35}{64} A^{2}+\frac{345}{256} B+\frac{315}{256} A B+\frac{1575}{2048} B^{2}\right) x^{2} \\
& +\left(\frac{23}{192}+\frac{15}{32} A+\frac{35}{384} A^{2}+\frac{185}{768} B+\frac{35}{192} A B+\frac{105}{1024} B^{2}\right) x^{3} \\
& -\left(\frac{65}{384}+\frac{11}{384} A+\frac{5}{768} A^{2}+\frac{35}{1536} B+\frac{35}{3072} A B+\frac{35}{6144} B^{2}\right) x^{4} \\
& +\left(\frac{1}{120}-\frac{B}{120}\right) x^{5}+2 e^{x}+\frac{1}{184320} e^{-2 x}((423360+705600 A \\
& \left.+378000 A^{2}+844200 B+1039500 A B+779625 B^{2}\right)+(349920 \\
& \left.+748800 A+453600 A^{2}+982800 B+1323000 A B+1039500 B^{2}\right) x \\
& +\left(119520+352800 A+252000 A^{2}+525600 B+793800 A B\right. \\
& \left.+661500 B^{2}\right) x^{2}+\left(20160+93120 A+84000 A^{2}+166800 B\right. \\
& \left.+294000 A B+264600 B^{2}\right) x^{3}+\left(1440+13920 A+18000 A^{2}\right. \\
& \left.+33480 B+73500 A B+73500 B^{2}\right) x^{4}+\left(960 A+2400 A^{2}\right. \\
& \left.+4080 B+12600 A B+14700 B^{2}\right) x^{5}+\left(160 A^{2}+240 B\right. \\
& \left.\left.\left.+1400 A B+2100 B^{2}\right) x^{6}+\left(80 A B+200 B^{2}\right) x^{7}+B^{2} x^{8}\right)\right) .
\end{aligned}
$$

If we expand $e^{x}$ and $e^{-2 x}$, the approximation of $u(x)$ is reduced to

$$
\begin{aligned}
u(x)= & 1+x+\frac{1}{2} x^{2}+\frac{1}{6} A x^{3}+\frac{1}{24} B x^{4}+\left(\frac{1}{60}-\frac{1}{120} B\right) x^{5}+\frac{1}{360} x^{6} \\
& +\frac{1}{2520} x^{7}+\left(\frac{1}{10080}-\frac{1}{20160} A\right) x^{8}-\left(\frac{1}{90720}-\frac{1}{45360} A\right. \\
& \left.+\frac{1}{181440} B\right) x^{9}+O\left(x^{9}\right) .
\end{aligned}
$$

Similarly, imposing the boundary conditions at $x=0$ and $x=1$ on $u(x)$ yields the values of $A$ and $B$, where

$$
A=1.031125908, B=0.7959257688 .
$$

Substituting (30) into (29) results in the approximate solution given by

$$
\begin{aligned}
u(x)= & 1+x+\frac{1}{2} x^{2}+0.171854318 x^{3}+0.0331635737 x^{4} \\
& +0.010033951927 x^{5}+\frac{1}{360} x^{6}+\frac{1}{2520} x^{7} \\
& +0.000048059231 x^{8}+7.322408858025 \times 10^{-6} x^{9}+O\left(x^{9}\right) .
\end{aligned}
$$


The comparisons between the errors obtained by using the homotopy perturbation method and by using the Sinc-Galerkin method [7] are given in Table 2. The numerical results clearly show that the homotopy perturbation method provides the approximate solutions obtained by two components of (25) in good agreement with the analytical solutions. Note that we obtain the approximate solutions without the Sinc interpolation which is inevitable when solving the fifth-order boundary value problems by using the Sinc-Galerkin method [7].

\section{Conclusions}

The homotopy perturbation method has been applied for solving the fifth-order boundary value problems. Compared with the sixth-degree B-spline function method, the Adomian decomposition method and Sinc-Galerkin method, the homotopy perturbation method provides a new approach to solve the fifthorder boundary value problems without discretization, Sinc interpolation and the computation of the Adomian polynomials and the coefficients of sixthdegree B-spline functions. Numerical results are presented to show the efficiency of the homotopy perturbation method. Therefore, this method can be seen as a promising and powerful tool for solving the the fifth-order boundary value problems.

Acknowledgement The author thank the anonymous referees for their discussions and comments.

\section{References}

[1] S. Abbasbandy, Application of He's homotopy perturbation method for Laplace transform, Chaos, Solitons \& Fractals, 30 (5) (2006), 1206-1212.

[2] R.P. Agarwal, Boundary Value Problems for High Ordinary Differential Equations, World Scientific, Singapore, 1986.

[3] H.N. Caglar, S.H. Caglar and E.H. Twizell, The numerical solution of fifth-order boundary value problems with sixth-degree B-spline functions, Appl. Math. Lett,12 (1999), 25-30.

[4] L. Cveticanin, Homotopy-perturbation method for pure nonlinear differential equation, Chaos, Solitons \& Fractals, 30 (5) (2006), 1221-1230.

[5] A.R. Davis, A. Karageorghis and T.N. Phillips, Spectral Galerkin methods for the primary two-point boundary-value problem in modeling viscoelastic flows, Internat. J. Numer. Methods Eng, 26 (1988), 647-662. 
[6] A.R. Davis, A. Karageorghis and T.N. Phillips, Spectral collocation methods for the primary two-point boundary-value problem in modelling viscoelastic flows, Internat. J. Numer. Methods Eng, 26 (1988), 805-813.

[7] M. El-Gamel, Sinc and the numerical solution of fifth-order boundary value problems, Appl. Math. Comput, (2006), doi:10.1016/j.amc.2006.09.049

[8] D.J. Fyfe, Linear dependence relations connecting equal interval Nth degree splines and their derivatives, J. Inst. Math. Appl, 7 (1971), 398-406.

[9] J.H. He, A coupling method of homotopy technique and perturbation technique for nonlinear problems, Internat. J. Non-linear Mech, 35 (1) (2000), 37-43.

[10] J.H. He, New interpretation of homotopy perturbation method, Internat. J. Modern Phys. B, 20 (18) (2006), 2561-2568.

[11] J.H. He, Homotopy perturbation technique, Comput. Math. Appl. Mech. Eng, 178 (3-4) (1999), 257-262.

[12] J.H. He, Homotopy perturbation method: A new nonlinear technique, Appl. Math. Comput, 135 (2003), 73-79.

[13] J.H. He, Limit cycle and bifurcation of nonlinear problems, Chaos, Solitons \& Fractals, 26 (2005), 827-833.

[14] J.H. He, Application of homotopy perturbation method to nonlinear wave equations, Chaos, Solitons \& Fractals, 26 (2005), 695-700.

[15] J.H. He, Periodic solutions and bifurcations of delay-differential equations, Phys. Lett. A, 347 (2005), 228-230.

[16] J.H. He, The homotopy-perturbation method for nonlinear oscillators with discontinuities, Appl. Math. Comput, 151 (2004), 287-292.

[17] J.H. He, Homotopy-perturbation method for bifurcation of nonlinear problems, Internat. J. Nonlinear Sci. Numer. Simul, 6 (2005), 207-208.

[18] M. A. Khan, Siraj-ul-Islam, I. A. Tirmizi, E.H. Twizell and S. Ashraf, A class of methods based on non-polynomial sextic spline functions for the solution of a special fifth-order boundary-value problems, J. Math. Anal. Appl, 321 (2006), 651-660.

[19] M.S. Khan, Finite-difference solutions of fifth-order boundary-value problems, Ph.D. Thesis, Brunel University, England, 1994. 
[20] S.J. Liao, An approximate solution technique not depending on small parameters: A special example, Internat. J. Non-Linear Mech, 30 (3) (1995), 371-380.

[21] M. Rafei and D.D. Ganji, Explicit solutions of Helmholtz equation and fifth-order KdV equation using homotopy perturbation method, Internat. J. Nonlinear Sci. Numer. Simul, 7 (3) (2006), 321-328.

[22] A.M. Siddiqui, R. Mahmood and Q.K. Ghori, Thin film flow of a third grade fluid on a moving belt by He??s homotopy perturbation method, Internat. J. Nonlinear Sci. Numer. Simul, 7(1) (2006), 7-14.

[23] A.M. Siddiqui, M. Ahmed and Q.K. Ghori, Couette and Poiseuille flows for non-Newtonian fluids, Internat. J. Nonlinear Sci. Numer. Simul, 7(1) (2006), 15-26.

[24] A. M. Wazwaz, The numerical solution of fifth-order boundary value problems by the decomposition method, J. Comput. Appl. Math, 136 (2001), 259-270.

Table 1. Comparisons of numerical errors

\begin{tabular}{cccc}
\hline $\mathrm{x}$ & Anal. solution & $u(x)[\mathrm{HPM}]$ & Errors[HPM] \\
\hline 0 & 0.0 & 0.0 & 0.0 \\
0.1571 & 0.15516533773 & 0.15516533773 & $2.8 E-17$ \\
0.3142 & 0.29881800212 & 0.29881800212 & $4.4 E-16$ \\
0.4712 & 0.41985059496 & 0.41985059496 & $3.2 E-14$ \\
0.6283 & 0.50831222097 & 0.50831222097 & $5.9 E-13$ \\
0.7854 & 0.55536064596 & 0.55536064597 & $5.1 E-12$ \\
0.9425 & 0.55397066969 & 0.55397066972 & $2.6 E-11$ \\
1.0996 & 0.49916624385 & 0.49916624394 & $8.5 E-11$ \\
1.2566 & 0.38835504690 & 0.38835504708 & $1.8 E-10$ \\
1.4137 & 0.22117471311 & 0.22117471328 & $1.7 E-10$ \\
$\frac{\pi}{2}$ & 0.0 & 0.0 & 0.0 \\
\hline
\end{tabular}


Table 2. Comparisons of numerical errors

\begin{tabular}{cccc}
\hline $\mathrm{x}$ & Anal. solution & Errors[HPM] & Errors[Sinc-Garlerkin] \\
\hline 0.0 & 1.0 & 0.0 & 0.0 \\
0.0100 & 1.010050167 & $1.2 E-9$ & 0.0 \\
0.1184 & 1.125694299 & $7.0 E-6$ & 0.0 \\
0.1517 & 1.163811041 & $1.4 E-5$ & $1.0 E-4$ \\
0.2410 & 1.272521035 & $4.6 E-5$ & 0.0 \\
0.3604 & 1.433902861 & $1.0 E-4$ & $1.0 E-4$ \\
0.4287 & 1.535260387 & $1.0 E-4$ & 0.0 \\
0.5000 & 1.648721271 & $1.9 E-4$ & $2.0 E-4$ \\
0.6395 & 1.895532876 & $1.0 E-4$ & $1.0 E-4$ \\
0.8482 & 2.335439276 & $9.8 E-5$ & $2.0 E-4$ \\
0.9996 & 2.717194733 & $1.2 E-9$ & $2.0 E-4$ \\
1.0 & 2.718281828 & 0.0 & 0.0 \\
\hline
\end{tabular}

Received: May 4, 2007 